\title{
Management of Guillain-Barré Syndrome
}

\author{
Kadarapura N. Gopalakrishna ${ }^{1}$ Venkatapura J. Ramesh ${ }^{1}$ \\ ${ }^{1}$ Department of Neuroanaesthesia and Neurocritical Care, National \\ Institute of Mental Health and Neurosciences (NIMHANS), \\ Bengaluru, Karnataka, India
}

\begin{abstract}
Address for correspondence Kadarapura N. Gopalakrishna, DNB, DM, Department of Neuroanaesthesia and Neurocritical Care, National Institute of Mental Health and Neurosciences (NIMHANS), Bengaluru 560 029, Karnataka, India (e-mail: gops868@gmail.com).
\end{abstract}

J Neuroanaesthesiol Crit Care 2019;6:160-166

\section{Introduction}

Guillain-Barré syndrome (GBS) is an acute or subacute onset, immune-mediated inflammatory disorder of the peripheral nervous system in which an autoimmune response is directed against peripheral nerves (myelin or axons) and spinal roots. ${ }^{1}$ The annual incidence rate of GBS across different studies range from approximately 0.5 to 2 cases per 100,000 people. ${ }^{2-4}$ The incidence rates increase with age, especially in those 60 years or older. There is also a gender difference; males are affected more than females (1.5:1). GBS is the most common cause of acute paralytic neuropathy. ${ }^{2,5}$ It usually presents with an acute and rapid progression of symmetric weakness of limbs, hyporeflexia or areflexia, and with or without involvement of respiratory muscles or cranial nerve. The majority of patients have an infection before the onset of GBS that is within 4 weeks, most commonly in the form of respiratory tract infection or gastroenteritis. ${ }^{6}$ The diagnosis of GBS is based on clinical features criteria supported by cerebrospinal fluid (CSF) findings and nerve conduction studies. The common subtypes of GBS based on clinical features, pathological, and electrophysiological findings are shown in $\boldsymbol{- T a b l e ~} \mathbf{1}{ }^{7}$ The complexity of interplay between infectious triggers and host factors determining the immune response shifting toward undesirable autoreactivity is unknown. ${ }^{8}$ The environmental and genetic factors contribution toward individuals vulnerable to develop the disease progression is unclear. ${ }^{9}$ GBS is most common paralytic neuropathy leading to respiratory failure, finally requiring mechanical ventilation. The poor prognostic factors include rapid progression, bulbar involvement, facial weakness (bilateral), and autonomic dysfunction..$^{10}$ The early specific standard treatment for GBS is plasma exchange (PE) or intravenous immunoglobulin (IVIg), and in addition, it requires supportive care and meticulous monitoring.

We searched PubMed and MEDLINE using the search term "Guillain Barre syndrome," and article published in English were included. This review article provides an overview of the etiopathophysiology, diagnostic criteria, and management of GBS.

\section{Pathophysiology}

The experimental evidence and clinical findings demonstrate that GBS is an immune-mediated disorder related to a complex synergistic interaction of humoral and cell-mediated immune received

February 11, 2019

accepted after revision

March 15, 2019

published online

June 3, 2019
DOI https://doi.org/

$10.1055 / \mathrm{s}-0039-1688896$

ISSN 2348-0548.
Copyright $\odot 2019$ Indian Society of Neuroanaesthesiology and Critical Care
License terms

() (1) $\ominus \circledast$ 
Table 1 Common subtypes of Guillain-Barré syndrome ${ }^{7}$

\begin{tabular}{|l|}
\hline $\begin{array}{l}\text { Acute inflammatory demyelinating polyradiculoneuropathy } \\
\text { (AIDP) }\end{array}$ \\
\hline Acute motor axonal neuropathy (AMAN) \\
\hline Acute motor sensory axonal neuropathy (AMSAN) \\
\hline Miller-Fisher syndrome (MFS) \\
\hline
\end{tabular}

response against the antigens of the peripheral nerve. ${ }^{11}$ Nearly $75 \%$ of patients report a preceding event, most commonly infection of the upper respiratory tract or gastrointestinal tract at least 1 to 4 weeks before the onset of GBS symptoms. ${ }^{4}$ The other triggers of GBS include vaccinations (including influenza virus vaccine, hepatitis vaccine, rabies vaccine, tetanus and diphtheria toxoids, and meningococcal vaccine), ${ }^{12}$ surgery, and trauma. ${ }^{13}$ The most common organism linked to GBS is Campylobacter jejuniinduced bacterial enteritis and is commonly linked to AMAN subtype. ${ }^{14}$ The other specific microbial agents, ${ }^{6}$ linked to GBS, include cytomegalovirus (CMV); hepatitis A, B, and E viruses ${ }^{15}$; Epstein-Barr virus, varicella zoster virus; Zika virus infection ${ }^{16}$; human immunodeficiency virus, Haemophilus influenzae ${ }^{17}$; and Mycoplasma pneumoniae. ${ }^{18}$ A preceding infection may result in an autoimmune response leading to production of antibodies against an infective agent that shares epitopes with the host's peripheral nerve gangliosides. This phenomenon is called postinfectious molecular mimicry. ${ }^{19}$ Currently, identification of the preceding infective trigger in a case of GBS may help in prognostication, developing individualized targeted management ${ }^{20}$ and preventing GBS cases in the region in future. ${ }^{21}$

\section{Diagnosis and Evaluation}

The classic form of GBS presents with rapidly progressive symmetrical weakness of the lower limbs. It ascends proximally over hours to few days and progresses to involve the upper limbs and facial, oropharyngeal, and respiratory muscles in severe cases. ${ }^{22}$ The diagnostic criteria for GBS were revised by Asbury and Cornblath in 1990 (-Table 2). ${ }^{23}$ The severity of illness varies from mild form in which patients are able to walk unassisted, to severe form leading to near-total quadriplegia. Sometimes, motor weakness develops simultaneously in all the four limbs or develops first in the arms followed by the legs. Most patients develop areflexia or hyporeflexia, but tendon reflex may be normal during early course of the disease. Cranial nerve involvement is common resulting in facial, extraocular, and bulbar weakness. ${ }^{7}$ In case of Miller-Fisher variant, typical clinical features include a triad of ophthalmoplegia, ataxia, and areflexia. ${ }^{24}$ Nearly $30 \%$ of GBS patients develop respiratory failure requiring endotracheal intubation and mechanical ventilation. ${ }^{24,25}$ Clinical findings suggesting impending respiratory muscle failure include tachypnea, tachycardia, sweating, unsynchronized thoracoabdominal movements, and use of accessory muscles of respiration. Apart from motor weakness, patients may have burning or tingling sensations of the limbs, ${ }^{26}$ pain in the extremities, ${ }^{27}$ sensory loss mainly impairment of vibration sense, and autonomic dysfunction. ${ }^{25}$ The autonomic
Table 2 Criteria for diagnosis of Guillain-Barré syndrome ${ }^{23}$

\begin{tabular}{|l|}
\hline Features required for GBS diagnosis \\
- Progressive motor weakness in the lower limbs and \\
- Areflexia \\
\hline Clinical features strongly supporting GBS diagnosis \\
\hline - Symptoms and signs progression over days, lasting up \\
to 4 wks \\
- Relative symmetry of symptoms and signs \\
- Mild sensory symptoms or signs \\
- other cranial nerve involvement (bilateral facial weakness or \\
- Autonomic dysfunction \\
- Recovery beginning $2-4$ wks after progression ceases \\
\hline CSF features strongly supportive of GBS diagnosis \\
\hline - Elevated CSF protein with cell count < 10 cells/ $\mu \mathrm{L}$ \\
\hline Electrodiagnostic features strongly supportive of GBS \\
diagnosis \\
\hline - Electrodiagnostic features of nerve conduction slowing \\
or block
\end{tabular}

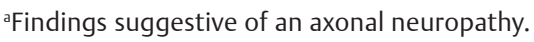

Abbreviations: CSF, cerebrospinal fluid; GBS, Guillain-Barré syndrome.

dysfunction may be present in the form of tachycardia or arrhythmias, hypertension, postural hypotension, sweating abnormalities, and bladder/bowel distubances. ${ }^{28}$ In addition, excessive vagal activity triggered by endotracheal suctioning or other stimulus can result in episodes of bradycardia, cardiac conduction block, or asystole. Clinically significant autonomic dysfunction may progress for up to 4 weeks of the illness. Cardiac arrhythmias in severe form with unstable hemodynamics is more often seen in patients with severe quadriparesis and respiratory failure. ${ }^{28}$ The diagnosis of GBS in young children is difficult because they present with atypical clinical features, and it is more difficult to perform neurological examination in them. ${ }^{29}$ In children, pain is the most common presentation before the motor weakness and leads to delay in the diagnosis of GBS. ${ }^{27}$ In view of a wide range of differential diagnosis in children, it is challenging to rule out other causes such as brainstem lesion, spinal cord lesion, muscular abnormalities, or other neuromuscular junction abnormalities. ${ }^{30}$

The diagnosis of GBS is supported by CSF examination and nerve conduction studies. The characteristic finding of CSF examination is the increased protein levels without pleocytosis, known as cytoalbuminologic dissociation. ${ }^{31}$ Usually in the first week of disease course the CSF may be normal, but thereafter from second week onward, there is an elevation in CSF protein in almost $90 \%$ of cases. ${ }^{25}$ Transient oligoclonal bands and increased myelin basic protein may be detected in the CSF of few GBS patients. ${ }^{7}$

Electrodiagnostic features of nerve conduction are usually normal in the early stage of the GBS course, but after 2 weeks of motor weakness onset, more than $90 \%$ of established GBS cases will have nerve conduction abnormalities. $^{32}$ Nerve conduction studies (NCSs) help distinguish 
acquired demyelination from axonal degeneration subtypes of GBS. In case of acute demyelinating polyneuropathy, NCS features include prolonged latency of distal motor, decreased conduction velocity of nerve, increased temporal dispersion, latency prolongation of F-wave, and nerve conduction blocks. In addition, sural nerve sensory response is often spared. ${ }^{33}$ The characteristic electrodiagnostic feature of acute motor axonal GBS is reduced amplitude or absent distal compound muscle action potential (CMAP). ${ }^{34} \mathrm{~A}$ few patients with axonal GBS will have slowing or temporary conduction blocks that usually recover rapidly during the course of the illness. This phenomenon is known as early reversible conduction failure. ${ }^{35}$ The transient feature of the reversible conduction failure initially may suggest acute inflammatory demyelinating polyradiculoneuropathy (AIDP), but it should be distinguished from axonal neuropathy by serial NCS over weeks. Reversible conduction failure specific to axonal neuropathy suggest that it is due to anti-ganglioside antibody-directed mechanism, and in future, targeted therapies can be developed. Electrodiagnostic studies might also predict prognosis, since patients with NCS features of demyelination often require mechanical ventilation, and decreased CMAP are more suggestive of poor outcome. Patients with axonal neuropathy may recover either very slowly or incompletely, or sometimes recover rapidly because of restoration of temporary conduction blocks. ${ }^{35}$

The anti-ganglioside antibodies levels can be used in the diagnosis of certain subtypes of GBS. Raised levels of anti-GQ1b ganglioside antibodies are found in more than $90 \%$ of Miller-Fisher syndrome (MFS) subtype of GBS. Preceding $C$. jejuni infection is linked to the AMAN variant and have elevated titers of anti-GM1, anti-GD1b, anti-GD1a, and anti-GalNAc-GD1a ganglioside antibodies of the IgG class. ${ }^{36}$ Raised serum antibodies to Mycoplasma, CMV, or C. jejuni can identify the preceding infection. Anti-galactocerebroside antibodies are found to be detected in patients with preceding Mycoplasma infection. ${ }^{\text {? }}$

Neuroimaging, especially magnetic resonance imaging (MRI) of the spine with contrast, has been used for diagnostic utility of GBS and also useful to rule out differential diagnostic abnormalities. Typical GBS has enhancement and swelling/thickening of the spinal nerve roots/cauda equina on spinal MRI. ${ }^{37,38}$

Other laboratory abnormalities in GBS include hyponatremia mostly in patients on mechanical ventilation, most likely due to inappropriate secretion of antidiuretic hormone, ${ }^{39}$ transient mild elevation in liver enzymes in almost about one-third of GBS patients without detectable cause, and rarely renal dysfunction due to glomerulonephritis (microscopic hematuria and proteinuria) because of immune complex deposition.

\section{Management}

The mainstay of treatment in GBS includes general supportive care and specific treatment with immunotherapy.

\section{General Supportive Care}

General supportive care is an important part of treatment of GBS. A multidisciplinary approach to prevent and manage potential complications in rapidly progressing GBS is important to reduce morbidity and mortality. ${ }^{28}$ Especially during the progressive phase of GBS, it is important to continuously monitor for respiratory failure, bulbar dysfunction, physical ability to handle secretions to prevent lung aspiration, and hemodynamics. The management of respiratory failure, hemodynamics, and cardiovascular dysfunction is most important and provides better chances for the favorable outcome. ${ }^{40}$ In addition, other issues to be addressed are deep vein thrombosis (DVT) prophylaxis, management of pain, treatment for possible impaired bowel and bladder function, autonomic dysfunction treatment, early physiotherapy initiation and plan for rehabilitation, and psychological support.

GBS patients with respiratory failure and bulbar dysfunction require mechanical ventilation. Predictors for the need of mechanical ventilation include rapid progression of disease course (onset of weakness to admission $<7$ days), severity of motor weakness, bulbar dysfunction, presence of neck and facial weakness, or dysautonomia. ${ }^{41,42}$ Predictors for endotracheal intubation and ventilatory assistance in GBS are shown in the - Table 3. ${ }^{1043,44}$ When the disease course strongly predicts for ventilatory support, tracheal intubation should be performed on an urgent basis before the crisis. Tracheostomy has to be performed when the ventilator support is longer than 2 weeks and there is no improvement in pulmonary function test from the baseline. If there is an improvement in strength and lung function test from baseline after 2 weeks, tracheostomy can be postponed for an additional week and meanwhile gradual weaning from the ventilator may be attempted. ${ }^{28}$ In centers experienced in percutaneous dilatation tracheostomy, it is found to be a superior method as compared with the traditional surgical tracheostomy in GBS patients. ${ }^{45}$ Weaning from mechanical ventilatory support should be based on improvement in strength and serial bedside lung function test. Patients requiring prolonged

Table 3 Predictors of intubation and ventilatory assistance in Guillain-Barré syndrome ${ }^{10,43,44}$

- Clinical evidence of inability to cough, unable to clear secretions, and neck weakness

- Signs of impending respiratory failure such as tachypnea, use of accessory muscles, inadequate chest rises, and paradoxical breathing with inadequate effort

- Simple bedside assessment tool

Single breath count $(S B C)<20$

Unable to complete the sentences

- Pulmonary function

Vital capacity $(V C)<15-20 \mathrm{~mL} / \mathrm{kg}$

Maximum expiratory pressure $\left(P_{\mathrm{F}} \max \right)<40 \mathrm{~cm} \mathrm{H} \mathrm{O}$

Maximum inspiratory pressure $\left(P_{1}\right.$ max $)<30 \mathrm{~cm} \mathrm{H}_{2} \mathrm{O}$

$>30 \%$ reduction in baseline VC, $P_{\mathrm{E}} \max$, and $P_{1}$ max

- Radiographic abnormalities include pulmonary infiltrates or atelectasis

- Arterial blood gas showing hypoxemia or hypercarbia or both 
mechanical ventilation are at an increased risk of ventilatory associated pneumonia, sepsis, bleeding from gastrointestinal tract, and other complications.

In view of marked fluctuations in blood pressure or cardiac arrhythmias in GBS, continuous blood pressure and electrocardiogram (ECG) monitoring helps in early identification and timely treatment. Extreme care should be taken in using antihypertensive or vasoactive agents in patients with dysautonomia. A painful stimulus such as endotracheal suction can trigger episodes of bradycardia and hypotension. Extra precaution should be taken during tracheal suction and to monitor oxygen saturation, heart rate, and blood pressure. The other autonomic disturbances such as hyponatremia, decreased mucosal function of bronchus, and adynamic ileus may be anticipated and managed accordingly.

The management of pain in GBS is extremely important during the whole spectrum of disease. During acute phase of the GBS, considerable decline in pain severity was noted for gabapentin use and also with the use of carbamazepine. ${ }^{46,47}$ Large, high-quality randomized controlled trials (RCTs) are required to prove the efficacy of gabapentin and carbamazepine for pain intervention in the acute and recovery phase of GBS. The other drugs used for neuropathic pain include tricyclic antidepressants, lidocaine, SSRI (selective norepinephrine and serotonin reuptake inhibitors), and topical capsaicin. ${ }^{48}$

Adequate high-energy nutrition with high caloric protein diet should be instituted early through enteral feeding. ${ }^{7}$ Nutrition plays an important role to prevent muscle wasting and helps in weaning patients from ventilatory support. In contrast to bolus feeding, continuous enteral feeding is better tolerated.

Subcutaneous unfractionated heparin or low-molecularweight heparin and calf compression devices should be used regularly in immobilized GBS patients to decrease the risk of DVT and pulmonary embolism. Prophylaxis for DVT should be continued until patients are able to walk with or without support. ${ }^{28}$

In the intensive care unit (ICU), pulmonary infection and urinary tract infection are common in patients with GBS. It is important to take preventive measures and aggressively treat nosocomial infections. Chest physiotherapy with tracheal suction and periodic change of patient position helps in preventing atelectasis in patients with depressed cough. Proficient nursing care with regular care of the mouth, skin, eyes, bowel, and bladder is necessary.

In case of facial weakness, exposure keratitis is prevented by supplementing with artificial tears and using adhesive tape to close the eyes at night. ${ }^{7}$ Position-related nerve palsies are prevented by adequate padding of pressure points. Physiotherapy of limbs started early in the disease course helps prevent joint contractures and immobilization. Constant reassurance and psychological support of patients and family are important for recovery.

\section{Immunotherapy}

The evidence-based proven therapies for GBS are PE and IVIg, and both have been shown to be equally effective. ${ }^{49-53}$

\section{Plasma Exchange}

Therapeutic PE has established benefit in acute GBS by decreasing the recovery time. PE initiated within 4 weeks of onset of weakness is proven beneficial in severe GBS, but substantial benefit was noted when started within 2 weeks. ${ }^{50,52,54,55}$ The recommended plasmapheresis includes a series of five PEs $(40-50 \mathrm{~mL} / \mathrm{kg})$ on alternate days. The therapeutic PE procedure involves removal of blood from the patient to separate inflammatory mediators such as pathogenic autoantibodies, cytokines, and complement components. The cellular components and some plasma along with replacement fluid such as crystalloid, albumin, or fresh frozen plasma are transfused to the patient. There are several techniques for plasmapheresis, which include filtration technique (membrane plasma separator) or centrifugation. ${ }^{56}$ Filtration technique usually requires a central venous access, and in this method, cellular elements are separated, but large molecules are able to pass through the filter. In centrifugation technique, the whole blood is spun until the components are separated and plasma components are removed in addition to immunoglobulins and soluble large molecules. Immunoadsorption technique is an alternative to PE in which plasma components are exposed to an absorptive material of variable selectivity, and thus only certain specific inflammatory mediators are removed rather the plasma itself. ${ }^{57}$

Based on moderate-quality evidence, PE improved the greater part of outcomes in comparison to supportive treatment alone. The time to regain strength to walk with support or without support and time to recover by 1 or 2 disability grades were decreased by PE. ${ }^{58} \mathrm{PE}$ reduced the time on ventilator, and the percentage of patients on ventilator support was significantly shortened. PE did not change the risk of cardiovascular instability or severe infections but definitely reduced the possibility of cardiac arrhythmias. Long-term benefits of PE after 1 year include significantly increased number of patients with fully recovered muscle strength and reduced the percentage of patients with severe motor deficits..$^{58}$

Based on the severity of disease, a trial suggested appropriate number of PE, which included two sessions for mild GBS and four sessions for moderate to severe GBS. ${ }^{59}$ There are no randomized studies that have assessed the efficacy of PE in children aged $<12$ years. ${ }^{58}$ The important considerations for using therapeutic $P E$ are venous access and requirement of high flow rates. The clotting factors depletion during PE and heparin use predisposes the subjects to risk of bleeding. ${ }^{60}$ Sometimes, citrate is used as an anticoagulant, which may predispose the patient to hypocalcemia or metabolic acidosis. Cost analyses estimated in different studies showed a decrease in the cost in patients with GBS treated with PE. ${ }^{61,62}$ Small-volume PE is one option, especially in developing countries where cost is the limiting factor. In the case of small-volume PE, $15 \mathrm{~mL} / \mathrm{kg} /$ day of volume of plasma is exchanged until the progression of disease is ceased or recovery started. ${ }^{63}$

\section{Intravenous Immunoglobulin}

The recommended regimen of IVIg for GBS is the total dose of $2 \mathrm{~g} / \mathrm{kg}$, that is, $0.4 \mathrm{~g} / \mathrm{kg} /$ day for 5 consecutive days. IVIg started 
within 2 weeks of onset of weakness accelerates recovery as good as PE. ${ }^{51,54}$ The main content of IVIg is IgG, and other molecules present are IgM, IgA, various cytokines, and soluble anti-TCR. ${ }^{56}$ These antibodies supplied by IVIg have the potential to bind and neutralize pathogenic antibodies. ${ }^{7}$ In many centers, IVIg has replaced therapeutic PE as the choice of treatment because of greater degree of accessibility, availability, and convenience of infusion at any time. There is some evidence that rapid infusion over 2 days had a better response compared with a longer course, ${ }^{64}$ but data from most trials show no such benefits..$^{52}$ Minor side effects of IVIg include myalgias, headache, arthralgias, flu-like symptoms, and fever. Minor infusion-related complications, though frequent, can often be prevented or controlled by slowing the rate of IVIg infusion or by symptomatic treatment. Sometimes serious complications such as anaphylaxis in IgA-deficient patients, congestive heart failure, transient renal dysfunction, and thrombotic complications are reported. ${ }^{65}$

\section{Corticosteroids}

Trials have demonstrated oral or intravenous corticosteroids alone in GBS have no clear benefits in the recovery or longterm effects. ${ }^{66-68}$ Though GBS is an inflammatory neuropathy due to autoimmune response, the reason for ineffectiveness of steroids is unknown.

\section{Combined Immunotherapy}

The effectiveness of the combined therapy of PE followed by IVIg showed no significant benefit when compared with PE or IVIg alone..$^{54,58}$ There are trials to show that combined therapy of IVIg and parenteral methylprednisolone had no clear benefit than IVIg alone. ${ }^{69,70}$

\section{Course and Prognosis}

About 5 to $15 \%$ of GBS-treated patients deteriorate after initial improvement or stable phase of the disease. Treatmentrelated fluctuations occur due to transient treatment effects of the prolonged disease course. In such scenario, IVIg treatment can be repeated, but there are no RCTs for assessing the utility of repeat IVIg course. By standard definition, patients should reach maximum neurological deficit within 1 month of onset of disease, if disease progresses for beyond 1 month, but less than 2 months is categorized as subacute or more than 2 months classified has chronic inflammatory demyelinating polyneuropathy (CIDP). Acute-onset CIDP is to be considered if GBS disease severity worsens after 2 months or have more than twice the treatment-related fluctuations. ${ }^{71}$ It is important to differentiate between acute-onset CIDP from recurrent GBS or GBS with treatment-related fluctuations, because acute-onset CIDP may require maintenance therapy or a change of treatment from IVIg to corticosteroids.

After disease progression stops, plateau phase lasts for 15 days or 1 month or longer before recovery begins. Most patients with GBS recover functionally, but still $20 \%$ of patients have residual motor deficits even after 1 year. Approximately $70 \%$ of patients will have complete recovery in 1 year and $82 \%$ in 2 years. ${ }^{7}$ Up to 2 to $7 \%$ of patients may have recurrence following complete recovery and seen commonly in young patients, mild GBS course, and MFS subtype. ${ }^{72,73}$ AIDP subtype of GBS has more recurrence than axonal subtypes. ${ }^{74}$ In general, prognosis of GBS is influenced by patients age, severity of illness at its peak, and dependence on whether immunotherapy started early. In addition, infectious episodes and acute hypoxic ischemia may worsen the prognosis. ${ }^{7}$

\section{Conclusion}

GBS is a common cause of acute paralytic polyneuropathy. Early course of the GBS requires meticulous monitoring and specific treatment with PE or IVIg. General supportive care, including management of respiratory failure, hemodynamics, and cardiovascular dysfunction, is most important and provides better chances for the favorable outcome.

\section{Conflict of Interest}

None declared.

\section{References}

1 Hughes RA, Cornblath DR. Guillain-Barré syndrome. Lancet 2005;366(9497):1653-1666

2 Sejvar J, Baughman AL, Wise M, Morgan OW. Population incidence of Guillain-Barré syndrome: a systematic review and meta-analysis. Neuroepidemiology 2011;36(2):123-133

3 Hallas J, Bredkjaer C, Friis ML. Guillain-Barré syndrome: diagnostic criteria, epidemiology, clinical course and prognosis. Acta Neurol Scand 1988;78(2):118-122

4 Govoni V, Granieri E. Epidemiology of the Guillain-Barré syndrome. Curr Opin Neurol 2001;14(5):605-613

5 Esposito S, Longo MR. Guillain-Barré syndrome. Autoimmun Rev 2017;16(1):96-101

6 Jacobs BC, Rothbarth PH, van der Meché FG, et al. The spectrum of antecedent infections in Guillain-Barré syndrome: a case-control study. Neurology 1998;51(4):1110-1115

7 Katirji B, Koontz D. Disorders of peripheral nerves. In: Robert BD, Gerald MF, Joseph J, John CM, eds. 6th ed. Bradley's Neurology in Clinical Practice. Philadelphia, PA: Elsevier Saunders; 2012:1955-1964

8 Willison HJ, Goodyear CS. Glycolipid antigens and autoantibodies in autoimmune neuropathies. Trends Immunol 2013;34(9):453-459

9 Blum S, McCombe PA. Genetics of Guillain-Barré syndrome (GBS) and chronic inflammatory demyelinating polyradiculoneuropathy (CIDP): current knowledge and future directions. J Peripher Nerv Syst 2014;19(2):88-103

10 Lawn ND, Fletcher DD, Henderson RD, Wolter TD, Wijdicks EF. Anticipating mechanical ventilation in Guillain-Barré syndrome. Arch Neurol 2001;58(6):893-898

11 Kieseier BC, Wiendl H, Hartung HP. The inflamed peripheral nervous syste update on immune therapies. Curr Opin Neurol 2006;19(5):433-436

12 Souayah N, Nasar A, Suri MF, Qureshi AI. Guillain-Barre syndrome after vaccination in United States a report from the CDC/FDA Vaccine Adverse Event Reporting System. Vaccine 2007;25(29):5253-5255

13 van Doorn PA, Ruts L, Jacobs BC. Clinical features, pathogenesis, and treatment of Guillain-Barré syndrome. Lancet Neurol 2008;7(10):939-950

14 Ho TW, Mishu B, Li CY, et al. Guillain-Barré syndrome in northern China. relationship to Campylobacter jejuni infection and anti-glycolipid antibodies. Brain 1995;118(Pt 3):597-605 
15 van den Berg B, van der Eijk A, Pas SD, et al. Guillain-Barré syndrome associated with preceding hepatitis $\mathrm{E}$ virus infection. Neurology 2014;82(6):491-497

16 Muñoz LS, Parra B, Pardo CA; Neuroviruses Emerging in the Americas Study. Neurological implications of Zika virus infection in Adults. J Infect Dis 2017;216(Suppl_10):S897-S905

17 Mori M, Kuwabara S, Miyake M, et al. Haemophilus influenzae infection and Guillain-Barré syndrome. Brain 2000;123(Pt 10): 2171-2178

18 Meyer Sauteur PM, Huizinga R, Tio-Gillen AP, et al. Mycoplasma pneumoniae triggering the Guillain-Barré syndrome: a case-control study. Ann Neurol 2016;80(4):566-580

19 Goodfellow JA, Willison HJ. Guillain-Barré syndrome: a century of progress. Nat Rev Neurol 2016;12(12):723-731

20 Doets AY, Jacobs BC, van Doorn PA. Advances in management of Guillain-Barré syndrome. Curr Opin Neurol 2018;31(5):541-550

21 Baker MG, Kvalsvig A, Zhang J, Lake R, Sears A, Wilson N. Declining Guillain-Barré syndrome after campylobacteriosis control, New Zealand, 1988-2010. Emerg Infect Dis 2012;18(2):226-233

22 Pithadia AB, Kakadia N. Guillain-Barré syndrome (GBS) Pharmacol Rep 2010;62(2):220-232

23 Asbury AK, Cornblath DR. Assessment of current diagnostic criteria for Guillain-Barré syndrome. Ann Neurol 1990;27(Suppl): S21-S24

24 van den Berg B, Walgaard C, Drenthen J, Fokke C, Jacobs BC, van Doorn PA. Guillain-Barré syndrome: pathogenesis, diagnosis, treatment and prognosis. Nat Rev Neurol 2014;10(8):469-482

25 Willison HJ, Jacobs BC, van Doorn PA. Guillain-Barré syndrome. Lancet 2016;388(10045):717-727

26 Moulin DE, Hagen N, Feasby TE, Amireh R, Hahn A. Pain in Guillain-Barré syndrome. Neurology 1997;48(2):328-331

27 Ruts L, Drenthen J, Jongen JL, et al; Dutch GBS Study Group. Pain in Guillain-Barré syndrome: a long-term follow-up study. Neurology 2010;75(16):1439-1447

28 Hughes RA, Wijdicks EF, Benson E, et al; Multidisciplinary Consensus Group. Supportive care for patients with Guillain-Barré syndrome. Arch Neurol 2005;62(8):1194-1198

29 Roodbol J, de Wit MC, Aarsen FK, Catsman-Berrevoets CE, Jacobs BC. Long-term outcome of Guillain-Barré syndrome in children. J Peripher Nerv Syst 2014;19(2):121-126

30 Eldar AH, Chapman J. Guillain Barré syndrome and other immune mediated neuropathies: diagnosis and classification. Autoimmun Rev 2014;13(4-5):525-530

31 Wong AH, Umapathi T, Nishimoto Y, Wang YZ, Chan YC, Yuki N. Cytoalbuminologic dissociation in Asian patients with Guillain-Barré and Miller Fisher syndromes. J Peripher Nerv Syst 2015;20(1):47-51

32 Hadden RD, Cornblath DR, Hughes RA, et al; Plasma Exchange/ Sandoglobulin Guillain-Barré Syndrome Trial Group. Electrophysiological classification of Guillain-Barré syndrome: clinical associations and outcome. Ann Neurol 1998;44(5):780-788

33 Vucic S, Cairns KD, Black KR, Chong PS, Cros D. Neurophysiologic findings in early acute inflammatory demyelinating polyradiculoneuropathy. Clin Neurophysiol 2004;115(10):2329-2335

34 Kokubun N, Nishibayashi M, Uncini A, Odaka M, Hirata K, Yuki $\mathrm{N}$. Conduction block in acute motor axonal neuropathy. Brain 2010;133(10):2897-2908

35 Kokubun N, Shahrizaila N, Hirata K, Yuki N. Reversible conduction failure is distinct from neurophysiological patterns of recovery in mild demyelinating Guillain-Barré syndrome. J Neurol Sci 2013;326(1-2):111-114

36 Jacobs BC, van Doorn PA, Schmitz PI, et al. Campylobacter jejuni infections and anti-GM1 antibodies in Guillain-Barré syndrome. Ann Neurol 1996;40(2):181-187

37 Galassi G, Genovese M, Ariatti A, Malagoli M. Early imaging in paraparetic Guillain-Barré syndrome. Acta Neurol Belg 2017
38 Berciano J, Gallardo E, Orizaola P, et al. Early axonal Guillain-Barré syndrome with normal peripheral conduction: imaging evidence for changes in proximal nerve segments. J Neurol Neurosurg Psychiatry 2016;87(5):563-565

39 Saifudheen K, Jose J, Gafoor VA, Musthafa M. Guillain-Barre syndrome and SIADH. Neurology 2011;76(8):701-704

40 Bosch EP. Guillain-Barre syndrome: an update of acute immune-mediated polyradiculoneuropathies. Neurologist 1998;4(11):211-226

41 Walgaard C, Lingsma HF, Ruts L, et al. Prediction of respiratory insufficiency in Guillain-Barré syndrome. Ann Neurol 2010;67(6):781-787

42 Green C, Baker T, Subramaniam A. Predictors of respiratory failure in patients with Guillain-Barré syndrome: a systematic review and meta-analysis. Med J Aust 2018;208(4):181-188

43 Sharshar T, Chevret S, Bourdain F, Raphaël JC; French Cooperative Group on Plasma Exchange in Guillain-Barré Syndrome. Early predictors of mechanical ventilation in Guillain-Barré syndrome. Crit Care Med 2003;31(1):278-283

44 Kannan Kanikannan MA, Durga P, Venigalla NK, Kandadai RM, Jabeen SA, Borgohain R. Simple bedside predictors of mechanical ventilation in patients with Guillain-Barre syndrome. J Crit Care 2014;29(2):219-223

45 Holdgaard HO, Pedersen J, Jensen RH, et al. Percutaneous dilatational tracheostomy versus conventional surgical tracheostomy. A clinical randomised study. Acta Anaesthesiol Scand 1998;42(5):545-550

46 Liu J, Wang LN, McNicol ED. Pharmacological treatment for pain in Guillain-Barre syndrome. Cochrane Database Syst Rev 2015;:CD009950

47 Pandey CK, Raza M, Tripathi M, Navkar DV, Kumar A, Singh UK. The comparative evaluation of gabapentin and carbamazepine for pain management in Guillain-Barré syndrome patients in the intensive care unit. Anesth Analg 2005;101(1):220-225 table of contents

48 Peña L, Moreno CB, Gutierrez-Alvarez AM. Pain management in Guillain-Barré syndrome: a systematic review. Neurologia 2015;30(7):433-438

49 van der Meché FG, Schmitz PI; Dutch Guillain-Barré Study Group. A randomized trial comparing intravenous immune globulin and plasma exchange in Guillain-Barré syndrome. N Engl J Med 1992;326(17):1123-1129

50 Plasmapheresis and acute Guillain-Barré syndrome. The Guillain-Barré syndrome Study Group. Neurology 1985;35(8):1096-1104

51 Hughes RA, Swan AV, van Doorn PA. Intravenous immunoglobulin for Guillain-Barré syndrome. Cochrane Database Syst Rev 2014;(9):CD002063

52 Hughes RA, Swan AV, Raphaël JC, Annane D, van Koningsveld R, van Doorn PA. Immunotherapy for Guillain-Barré syndrome: a systematic review. Brain 2007;130(Pt 9):2245-2257

53 Hughes RA. Plasma exchange versus intravenous immunoglobulin for Guillain-Barré syndrome. Ther Apher 1997;1(2):129-130

54 Plasma Exchange/Sandoglobulin Guillain-Barré Syndrome Trial Group. Randomised trial of plasma exchange, intravenous immunoglobulin, and combined treatments in Guillain-Barré syndrome. Lancet 1997;349(9047):225-230

55 Raphaël JC, Chevret S, Hughes RA, Annane D. Plasma exchange for Guillain-Barré syndrome. Cochrane Database Syst Rev 2012;(7):CD001798

56 Mitchell CW, Bertorini TE. Principles and Guidelines of Immunotherapy in Neuromuscular Disorders. In: Bertorini TE, ed. Neuromuscular Disorders: Treatment and Management. Philadelphia, PA: Saunders Elsevier; 2011:101-113

57 Marn Pernat A, Buturović-Ponikvar J, Svigelj V, Ponikvar R. Guillain-Barré syndrome treated by membrane plasma exchange and/or immunoadsorption. Ther Apher Dial 2009;13(4):310-313 
58 Chevret S, Hughes RA, Annane D. Plasma exchange for Guillain-Barré syndrome. Cochrane Database Syst Rev 2017;2:CD001798

59 The French Cooperative Group on Plasma Exchange in Guillain-Barré Syndrome. Appropriate number of plasma exchanges in Guillain-Barré syndrome. Ann Neurol 1997;41(3):298-306

60 Lehmann HC, Hartung HP, Hetzel GR, Stüve O, Kieseier BC. Plasma exchange in neuroimmunological disorders: Part 1: rationale and treatment of inflammatory central nervous system disorders. Arch Neurol 2006;63(7):930-935

61 Espérou H, Jars-Guincestre MC, Bolgert F, Raphaël JC, Durand-Zaleski I; French Cooperative Group on Plasma Exchange in Guillain-Barré Syndrome. Cost analysis of plasma-exchange therapy for the treatment of Guillain-Barré syndrome. Intensive Care Med 2000;26(8):1094-1100

62 Osterman PO, Fagius J, Lundemo G, et al. Beneficial effects of plasma exchange in acute inflammatory polyradiculoneuropathy. Lancet 1984;2(8415):1296-1299

63 Tharakan J, Jayaprakash PA, Iyer VP. Small volume plasma exchange in Guillain-Barre syndrome: experience in 25 patients. J Assoc Physicians India 1990;38(8):550-553

64 Dalakas MC. The use of intravenous immunoglobulin in the treatment of autoimmune neuromuscular diseases: evidence-based indications and safety profile. Pharmacol Ther 2004;102(3):177-193

65 Brannagan TH III. Intravenous gamma globulin (IVIg) for treatment of CIDP and related immune-mediated neuropathies. Neurology 2002;59(12 (Suppl 6):S33-S40

66 Guillain-Barré Syndrome Steroid Trial Group. Double-blind trial of intravenous methylprednisolone in Guillain-Barré syndrome. Lancet 1993;341(8845):586-590
67 Hughes RA, Swan AV, van Doorn PA. Corticosteroids for Guillain-Barré syndrome. Cochrane Database Syst Rev 2010; (2):CD001446

68 Hughes RA, Brassington R, Gunn A, van Doorn PA. Corticosteroids for Guillain-Barré syndrome. Cochrane Database Syst Rev 2016;10:CD001446

69 Susuki K, Yuki N. Effect of methylprednisolone in patients with Guillain-Barré syndrome. Lancet 2004;363(9416):1236-1237, author reply 1237-1238

70 van Koningsveld R, Schmitz PI, Meché FG, Visser LH, Meulstee J, van Doorn PA; Dutch GBS study group. Effect of methylprednisolone when added to standard treatment with intravenous immunoglobulin for Guillain-Barré syndrome: randomised trial. Lancet 2004;363(9404):192-196

71 Ruts L, Drenthen J, Jacobs BC, van Doorn PA; Dutch GBS Study Group. Distinguishing acute-onset CIDP from fluctuating Guillain-Barre syndrome: a prospective study. Neurology 2010;74(21):1680-1686

72 Kuitwaard K, Bos-Eyssen ME, Blomkwist-Markens PH, van Doorn PA. Recurrences, vaccinations and long-term symptoms in GBS and CIDP. J Peripher Nerv Syst 2009;14(4):310-315

73 Ishii J, Yuki N, Kawamoto M, Yoshimura H, Kusunoki S, Kohara N. Recurrent Guillain-Barré syndrome, Miller Fisher syndrome and Bickerstaff brainstem encephalitis. J Neurol Sci 2016;364:59-64

74 Notturno F, Kokubun N, Sekiguki Y, et al. Demyelinating Guillain-Barré syndrome recurs more frequently than axonal subtypes. J Neurol Sci 2016;365:132-136 